\title{
Local Uniqueness in the Cauchy Problem for Second Order Elliptic Equations with Non-Lipschitzian Coefficients
}

By

\author{
Shigeo TARAMA*
}

\begin{abstract}
We show the local uniqueness of the Cauchy problem for the second order elliptic operators whose coefficients of the principal part are real-valued and continuous with some modulus of continuity. These coefficients are not necessarily lipschitz continuous. The proof is given by drawing the Carleman estimates with a weight attached to the modulus of continuity.
\end{abstract}

\section{§1. Introduction}

For the second order elliptic operator with real coefficients, the local uniqueness in the Cauchy problem holds if the coefficients of second order terms are lipschitz continuous (see L. Hörmander [H1] or [H2]). On the other hand, according to the example of A. Plis ([P]), if the coefficients are only Hölder continuous with the index strictly smaller than 1 , the assertion above dose not hold in general. In this paper we introduce some class of continuous functions which contains some non-lipschitz continuous functions and show that if all the coefficients of second order terms belong to this class, the local uniqueness holds.

In order to define a class of continuous functions, we first introduce a function by which we measure the modulus of continuity. We assume that the positive and nondecreasing continuous function $\chi(s)$ defined on the interval $[1,+\infty)$ satisfies the following: there exists a constant $C>0$ such that on $[1,+\infty)$

$$
\chi(2 s) \leq C \chi(s)
$$

Communicated by T. Kawai, March 27, 1996. Revised July 24, 1996.

1991 Mathematics Subject Classification(s): 35B05, 35J15

* Dep. Appl. Mathematics and Physics, Fac. of Engineering, Kyoto University 606-01 Kyoto, Japan 
next

$$
\int_{1}^{+\infty} \frac{1}{s \chi(s)} d s=+\infty
$$

and finally with some constants $\delta \in(0,1)$ and $C>0$

$$
|\chi(s)| \leq C s^{\delta} \quad \text { for } s \geq 1
$$

Remark. We see that, by choosing large positive constants $C_{1}, C_{2}$ and $C_{3}$, the functions $\log \left(C_{1} s\right)$ and $\log \left(C_{2} s\right) \log \left(\log \left(C_{3} s\right)\right.$ satisfy (M.1), (M.2) and (M.3).

Using the positive and nondecreasing continuous function $\chi(r)$ that satisfies (M.1), (M.2) and (M.3), we define a function space $C^{x}$. Let $\Omega$ be an open set in $\boldsymbol{R}^{d+1}$. We denote by $C^{\chi}(\Omega)$ the space of all continuous $f(x)$ on $\Omega$ satisfying that for any compact set $K$ in $\Omega$, there exist two constants $\epsilon \in(0,1]$ and $C>0$ such that for any $x, y \in K$ satisfying $|x-y| \leq \epsilon$,

$$
|f(x)-f(y)| \leq C|x-y| \chi\left(\frac{1}{|x-y|}\right) .
$$

Let $\Omega$ be an open set in $\boldsymbol{R}^{d+1}$. We consider an elliptic operator $E\left(x, D_{x}\right)$ defined by

$$
E\left(x, D_{x}\right) u(x)=\sum_{j, k=0}^{d} a_{j, k}(x) D_{x_{j}} D_{x_{k}} u(x)+\sum_{j=0}^{d} a_{j}(x) D_{x_{j}} u(x)+c(x) u(x)
$$

where $D_{x_{j}}=\frac{1}{i} \frac{\partial}{\partial x_{j}}$ and the coefficients satisfy the following;

The coefficients $a_{j . k}(x)$ of second order terms are real-valued and belong to $C^{x}(\Omega)$. Furthermore, for any compact set $K$ of $\Omega$, there exists a positive constant $C_{K}$ such that

$$
\sum_{j, k=0}^{d} a_{j, k}(x) \xi_{j} \xi_{k} \geq C_{K}|\xi|^{2}
$$

for any $x \in K$ and any $\left(\xi_{0}, \cdots, \xi_{d}\right) \in \mathbb{R}^{d+1}$. And

$$
a_{j}(x), c(x) \in L_{l o c}^{\infty}(\Omega),
$$

that is to say, they are locally essentially bounded.

Under the assumptions above we have the following; 
Theorem 1.1. Let $x^{0}$ be a point of $\Omega, V$ a neighborhood of $x^{0}$ and $N$ a hypersurface in $V$ defined by $\{x \in V \mid \theta(x)=0\}$ where $\theta(x) \in C^{2}(V)$ satisfies $\nabla \theta(x) \neq 0$ and $\theta\left(x^{0}\right)=0$. Then there exists an open neighborhood $W$ of $x^{0}$ such that for any $u(x) \in H^{2}(V)$ that vanishes on $\{x \in V \mid \theta(x)<0\}$ and satisfies on $V$

$$
E\left(x, D_{x}\right) u(x)=0
$$

we have $u(x)=0$ on $W$.

Here we denote by $H^{2}(V)$, where $V$ is an open set $\subset \boldsymbol{R}_{x}^{d+1}$, the space that consists of all $u(x)$ in $L^{2}(V)$ whose first and second order derivatives also belong to $L^{2}(V)$, where $L^{2}(V)$ is the space of square integrable functions on $V$.

By a $C^{2}$-change of coordinates, we may assume that $\theta(x)=x_{0}$ and $x^{0}=0$. Note that the function classes $H_{l o c}^{2}(V)$, that is a space of all $f(x)$ satisfying $\epsilon(x) f(x) \in H^{2}(V)$ for any compactly supported smooth $\epsilon(x)$ on $V$, and $C^{\chi}$ are invariant under any $C^{2}$-change of coordinates. Furthermore, Holmgren's transformation (see L. Nirenberg [Ni, §7, page 29 and 31] or C. Zuily [Z, page 43]) implies that in order to prove Theorem 1.1 we have only to prove the following Theorem 1.2. Indeed after Holmgren's transformation, we may assume that there exist an open neighborhood $V$ of the origin and $t_{0}>0$ such that $u(x) \in H^{2}(V), E\left(x, D_{x}\right) u(x)=0$ in $V$ and $u(x)=0$ on $V \cap\left\{\left(x_{0}, x^{\prime}\right)\right.$ $\in \boldsymbol{R}^{d+1} \mid x_{0}<t_{0}$ and $\left.x_{0}-\left|x^{\prime}\right|^{2}<0\right\}$ where $\left\{\left.\left(x_{0}, x^{\prime}\right) \in \boldsymbol{R}^{d+1}|| x_{0}\left|<t_{0},\right| x^{\prime}\right|^{2}<t_{0}\right\}$ is relatively compact in $V$. Thus, since $h\left(x_{0}\right) u(x)$, where $h\left(x_{0}\right) \in C^{\infty}(\boldsymbol{R})$ verifies $h\left(x_{0}\right)=0$ for $\left[\frac{2}{3} t_{0},+\infty\right)$ and $h\left(x_{0}\right)=1$ on $\left(-\infty, \frac{1}{2} t_{0}\right]$, satisfies, by setting $t=x_{0}$ and $x=x^{\prime}$, the assumption of Theorem 1.2, we see $u(x)=0$ for $x_{0}<\frac{1}{3} t_{0}$.

Theorem 1.2. Let $E\left(t, x, D_{t}, D_{x}\right)$ be a second order elliptic operator on $\boldsymbol{R}_{t} \times \boldsymbol{R}_{x}^{d}$ defined by

$$
\begin{array}{r}
E\left(t, x, D_{t}, D_{x}\right)=D_{t}^{2}+2 \sum_{j=1}^{d} a_{0, j}(t, x) D_{t} D_{x_{j}}+\sum_{j, k=1}^{d} a_{j, k}(t, x) D_{x_{j}} D_{x_{k}} \\
+a_{0}(t, x) D_{t}+\sum_{j=1}^{d} a_{j}(t, x) D_{x_{j}}+c(t, x)
\end{array}
$$

where the coefficients satisfy the following;

(1) All the coefficients are constant outside some compact set $K$ in $\boldsymbol{R}^{d+1}$.

(2) For $1 \leq j, k \leq n, a_{0, j}(t, x)$ and $a_{j, k}(t, x)$ are real-valued and belong to $C^{\chi}\left(\boldsymbol{R}^{d+1}\right)$. 
(3) $a_{0}(t, x), a_{j}(t, x)$ for $j=1, \cdots, d$ and $c(t, x)$ are in $L^{\infty}\left(\boldsymbol{R}^{d+1}\right)$

(4) There exists a constant $C>0$ such that for any $\tau \in \boldsymbol{R}$, any $\left(\xi_{1}, \cdots, \xi_{d}\right) \in \boldsymbol{R}^{d}$ and any $(t, x) \in \boldsymbol{R}^{d+1}$

$$
\tau^{2}+2 \sum_{j=1}^{d} a_{0, j}(t, x) \tau \xi_{j}+\sum_{j, k=1}^{d} a_{j, k}(t, x) \xi_{j} \xi_{k} \geq C\left(|\tau|^{2}+|\xi|^{2}\right) .
$$

Let $t_{0}$ be a positive number. Assume that $u \in H^{2}\left(\boldsymbol{R}^{d+1}\right)$ whose support is contained in $\left[0, \frac{2}{3} t_{0}\right] \times \boldsymbol{R}_{x}^{d}$ satisfies

$$
E\left(t, x, D_{t}, D_{x}\right) u(t, x)=f(t, x)
$$

where $f(t, z) \in L^{2}\left(\boldsymbol{R}^{d+1}\right)$ vanishes for $t<\frac{1}{2} t_{0}$. Then we have $u(t, x)=0$ for $t<\frac{1}{3} t_{0}$.

Here $L^{\infty}\left(\boldsymbol{R}^{d+1}\right)$ is the space of all essentially bounded functions on $\boldsymbol{R}^{d+1}$. The proof of Theorem 1.2 is given in Section 4 after two preparations. First, in the next section, we define the regularization of functions in $C^{\chi}$ and show their properties. As for the second one we draw the Carleman estimates for some elliptic first order equations in Section 3.

Remark. The function class $C^{\chi}$ is studied by H. Bahouri and J.-Y. Chemin ([B-C]) in the context of fluid dynamics. Furthermore F. Colombini and N. Lerner ([C-L]) studied the Cauchy problem for second order strictly hyperbolic operators with log-lipschitz continuous coefficients, that is to say belonging to $C^{\chi}$ with $\chi(r)=\log (2 r)$.

In the following sections, we use the notation of the multi-index $\alpha$ which is a $d$-tuple of non-negative integers $\left(\alpha_{1}, \cdots, \alpha_{d}\right)$. We set $|\alpha|=\sum_{j=1}^{d} \alpha_{j}$. The space $C_{0}^{\infty}\left(\boldsymbol{R}^{d}\right)$ is the space of all compactly supported smooth functions on $\boldsymbol{R}^{d}$. We denote by $\|\cdot\|[$ resp. $\|\cdot \cdot\|]$ the $L^{2}$-norm in $\boldsymbol{R}_{x}^{d}\left[\right.$ resp. $\boldsymbol{R}_{t} \times \boldsymbol{R}_{x}^{d}$ that is to say;

$$
\|f(x)\|^{2}=\int_{R^{d}}|f(x)|^{2} d x
$$

[resp.

$$
\left.\left.\|f(t, x)\|\right|^{2}=\int_{\boldsymbol{R}^{d+1}}|f(t, x)|^{2} d t d x .\right]
$$

Set $\langle\xi\rangle=\sqrt{|\xi|^{2}+1}$.

For a symbol $a(t, x, \xi)$, which does not necessarily depend on $t$ or $x$, we 
denote by $a\left(t, x, D_{x}\right)$ the pseudodifferential operator defined by

$$
a\left(t, x, D_{x}\right) f(x)=\frac{1}{(2 \pi)^{d}} \int_{\mathbf{R}^{\boldsymbol{d}}} e^{i\langle x, \xi\rangle} a(t, x, \xi) \hat{f}(\xi) d \xi
$$

where $\hat{f}(\xi)$ is the Fourier transform of $f(x)$, that is to say

$$
\hat{f}(\xi)=\int_{\boldsymbol{R}^{d}} e^{-i\langle\xi, x\rangle} f(x) d x .
$$

Furthermore we denote by $C$ or suffixed $C_{*}$ some positive constant which may be different line by line.

\section{§2. Preliminaries}

In this section, we define the regularization of functions in $C^{x}$ and show its properties.

Let a nonnegative and nonincreasing function $\Psi(y) \in C^{\infty}(R)$ satisfy

$$
\begin{gathered}
0 \leq \Psi(y) \leq 1, \\
\Psi(y)= \begin{cases}1, & \text { for } y \leq 1 \\
0, & \text { for } y \leq 2 .\end{cases}
\end{gathered}
$$

We define a sequence of functions $\left\{\psi_{j}(y)\right\}$ in the following way:

$$
\begin{aligned}
& \psi_{0}(y)=\Psi(y) \\
& \psi_{j}(y)=\Psi\left(2^{-j} y\right)-\Psi\left(2^{-j+1} y\right) \text { for } j \geq 1 .
\end{aligned}
$$

Then we see that, for $j \geq 1$, the support of $\psi_{j}(y)$ is contained in $\left\{y \mid 2^{j-1} \leq y \leq 2^{j+1}\right\}$ and that

$$
\left|\frac{d^{k}}{d y^{k}} \psi_{j}(y)\right| \leq C 2^{-j k}
$$

where the constant $C$ may depend on $k$.

For a positive and nondecreasing continuous function $\chi(s)$ satisfying (M.1), (M.2) and (M.3), we define a nondecreasing function $\tilde{\chi}(s)$ by

$$
\tilde{\chi}(s)=\sum_{j=0}^{+\infty} \psi_{j}(s) \chi\left(2^{j}\right)
$$


The property (M.1) implies that

$$
C^{-1} \leq \frac{\tilde{\chi}(s)}{\chi(s)} \leq C \text { on }[1,+\infty)
$$

which shows that $C^{\chi}=C^{\tilde{\chi}}$. We see also that $\tilde{\chi}(s)$ satisfies (M.2) and (M.3). Furthermore from (2.1) we obtain

$$
\left|\frac{d^{n}}{d s^{n}} \tilde{\chi}(s)\right| \leq C(1+|s|)^{-n} \tilde{\chi}(s)
$$

where the constant $C$ may depend on $n$.

Therefore from now on we assume that the function $\chi(s)$ is a positive and nondecreasing smooth function enjoying the properties (M.1), (M.2), (M.3) and (M.4).

Next we define the regularization $\tilde{a}(t, x, \xi)$ of a function $a(t, x) \in C^{\chi}\left(\boldsymbol{R}^{d+1}\right)$ by using the functions above $\psi_{j}(y)$ and a nonnegative function $\gamma(w) \in C_{0}^{\infty}\left(\boldsymbol{R}^{d+1}\right)$ satisfying

$$
\int_{\mathbf{R}^{d+1}} \gamma(w) d w=1
$$

and

$$
\gamma(w)=0 \quad \text { if }|w|<1 \text { or }|w|>2 .
$$

Set

$$
\rho_{j}=\left(2^{j} \chi\left(2^{j}\right)\right)^{\frac{1}{2}}
$$

We define $\tilde{a}(t, x, \xi)$ by

$$
\tilde{a}(t, x, \xi)=\sum_{j=0}^{+\infty} \psi_{j}(|\xi|) \rho_{j}^{d+1} \int_{R^{d+1}} \gamma\left(\rho_{j}(t-s), \rho_{j}(x-y)\right) a(s, y) d s d y
$$

Then we see the following:

Proposition 2.1. For any $a(t, x) \in C^{x}\left(\boldsymbol{R}^{d+1}\right)$ that is constant out of a compact set, we have the following:

$$
|\tilde{a}(t, x, \xi)-a(t, x)| \leq C\langle\xi\rangle^{-\frac{1}{2}}(\chi(\langle\xi\rangle))^{\frac{1}{2}}
$$


and for $\alpha_{0}+|\alpha|+|\beta|>0$

$$
\begin{aligned}
& \left|\frac{\partial^{\alpha_{0}}}{\partial t^{\alpha_{0}}} \frac{\partial^{\alpha}}{\partial x^{\alpha}} \frac{\partial^{\beta}}{\partial \xi^{\beta}} \tilde{a}(t, x, \xi)\right| \\
& \quad \leq C\langle\xi\rangle^{\frac{\left|\alpha_{0}\right|+|\alpha|-1}{2}}(\chi(\langle\xi\rangle))^{\frac{\left|\alpha_{0}\right|+|\alpha|+1}{2}}\langle\xi\rangle^{-|\beta|}
\end{aligned}
$$

And the operator

$$
\left(\tilde{a}\left(t, x, D_{x}\right)-a(t, x)\right)\left\langle D_{x}\right\rangle^{\frac{1}{2}}\left(\chi\left(\left\langle D_{x}\right\rangle\right)\right)^{-\frac{1}{2}}
$$

is an $L^{2}$ bounded operator and strongly continuous with respect to $t$.

Proof. In this proof $C$ represents an arbitrary constant which is independent of $j$. Since $a(t, x) \in C^{\chi}\left(\boldsymbol{R}^{d+1}\right), \chi(s)$ is nondecreasing and $\rho_{j} \leq 2^{j}$ for large $j$, which follows from (2.2) and (M.3), then we see that, if $\left(\rho_{j}(s-t), \rho_{j}(y-x)\right) \in \operatorname{supp} \gamma(w)$, then

$$
\begin{aligned}
|a(s, y)-a(t, x)| & \leq C \rho_{j}^{-1} \chi\left(\rho_{j}\right) \\
& \leq C\left(2^{-j} \chi\left(2^{j}\right)\right)^{\frac{1}{2}},
\end{aligned}
$$

where we used the assumption that $a(t, x)$ is constant out of a compact set and $\chi\left(\rho_{j}\right) \leq C \chi\left(2^{j}\right)$ which follows from (M.1) and from $\rho_{j} \leq 2^{j}$ for large $j$. Thus we see (2.3).

Next we remark that the estimate (2.1) implies, for $j \geq 1$,

$$
\left|\frac{\partial^{\beta}}{\partial \xi^{\beta}} \psi_{j}(|\xi|)\right| \begin{cases}\leq C\langle\xi\rangle^{-|\beta|} & \text { on }\left\{\xi\left|2^{j-1} \leq\right| \xi \mid \leq 2^{j+1}\right\} \\ =0 & \text { otherwise. }\end{cases}
$$

If $\left|\alpha_{0}\right|+|\alpha|>0$, since $\int \frac{\partial^{\alpha_{0}}}{\partial s^{\alpha_{0}}} \frac{\partial^{\alpha}}{\partial y^{\alpha}} \gamma(s, y) d s d y=0$, we have

$$
\begin{aligned}
& \int_{\mathbf{R}^{d+1}}(\left(\frac{\partial^{\alpha_{0}}}{\partial s^{\alpha_{0}}} \frac{\partial^{\alpha}}{\partial y^{\alpha}} \gamma\left(\rho_{j}(t-s), \rho_{j}(x-y)\right)\right) a(s, y) d s d y \\
& \quad=\int_{\boldsymbol{R}^{d+1}}\left(\frac{\partial^{\alpha_{0}}}{\partial s^{\alpha_{0}}} \frac{\partial^{\alpha}}{\partial y^{\alpha}} \gamma\left(\rho_{j}(t-s), \rho_{j}(x-y)\right)\right)(a(s, y)-a(t, x)) d s d y .
\end{aligned}
$$

From (2.5) we see that the absolute value of the right hand side of the equation above is equal or inferior to $C \rho_{j}^{-(d+2)} \chi\left(\rho_{j}\right)$. Since $\chi\left(\rho_{j}\right) \leq C \chi\left(2^{j}\right)$ and $\left|\alpha_{0}\right|+|\alpha|>0$, 
using (2.2), we get

$$
\rho_{j}^{\left|\alpha_{0}\right|+|\alpha|} \rho_{j}^{-1} \chi\left(\rho_{j}\right) \leq C\left(2^{\frac{1}{2} j}\right)^{\left|\alpha_{0}\right|+|\alpha|-1}\left(\chi\left(2^{j}\right)\right)^{\left|\alpha_{0}\right|+|\alpha|+1},
$$

from which and from (2.6) follows the estimate (2.4) for $\left|\alpha_{0}\right|+|\alpha|>0$. In the case where $|\beta|>0$ and $\left|\alpha_{0}\right|+|\alpha|=0$, since $\Sigma_{j=0}^{+\infty} \psi_{j}(|\xi|)=1$ and $\rho_{j}^{d+1} \int \gamma\left(\rho_{j}(t-s)\right.$, $\left.\rho_{j}(x-y)\right) d s d y=1$, we see

$$
\frac{\partial^{\beta}}{\partial \xi^{\beta}} \tilde{a}(t, x, \xi)=\sum_{j=0}^{+\infty} \frac{\partial^{\beta}}{\partial \xi^{\beta}} \psi_{j}(|\xi|) \rho_{j}^{d+1} \int_{R^{d+1}} \gamma\left(\rho_{j}(t-s), \rho_{j}(x-y)\right)(a(s, y)-a(t, x)) d s d y,
$$

from which and (2.5) we see the estimate of (2.4) in this case.

Finally we show the $L^{2}$-boundedness of the operator

$$
\left(\tilde{a}\left(t, x, D_{x}\right)-a(t, x)\right)\left\langle D_{x}\right\rangle^{\frac{1}{2}}\left(\chi\left(\left\langle D_{x}\right\rangle\right)\right)^{-\frac{1}{2}}
$$

by using the method of $\mathrm{M}$. Nagase ([Na]).

Put

$$
A(t, x, \xi)=(\tilde{a}(t, x, \xi)-a(t, x))\langle\xi\rangle^{\frac{1}{2}}(\chi(\langle\xi\rangle))^{-\frac{1}{2}}
$$

Then we see from (2.3), (2.4) and (M.4)

$$
\left|\frac{\partial^{\beta}}{\partial \xi^{\beta}} A(t, x, \xi)\right| \leq C\langle\xi\rangle^{-|\beta|}
$$

On the other hand we obtain from (M.4), for any $\beta$,

$$
\begin{aligned}
& \left|\frac{\partial^{\beta}}{\partial \xi^{\beta}}\left(A\left(t, x_{1}, \xi\right)-A\left(t, x_{2}, \xi\right)\right)\right| \\
& \quad \leq C\left|x_{1}-x_{2}\right| \chi\left(\left|x_{1}-x_{2}\right|^{-1}\right)\langle\xi\rangle^{\frac{1}{2}}(\chi(\langle\xi\rangle))^{-\frac{1}{2}}\langle\xi\rangle^{-|\beta|}
\end{aligned}
$$

Therefore for the symbol $\tilde{A}(t, x, \xi)$ defined by

$$
\tilde{A}(t, x, \xi)=\langle\xi\rangle^{d \mu} \int_{\mathbf{R}^{d}} \gamma_{1}\left(\langle\xi\rangle^{\mu}(x-y)\right) A(t, y, \xi) d y
$$

where $\mu=\frac{\delta+2}{3}$ with the constant $\delta$ in (M.3) and a function $\gamma_{1}(y) \in C_{0}^{\infty}\left(\boldsymbol{R}^{d}\right)$ satisfies $\gamma_{1}(y)=0$ out of $\{y|1 \leq| y \mid \leq 2\}$ and

$$
\int_{\mathbf{R}^{d}} \gamma_{1}(y) d y=1
$$


we have the following; since we see, from $0<\mu<1, \chi\left(\langle\xi\rangle^{\mu}\right) \leq \chi(\langle\xi\rangle)$, then for any $\beta$

$$
\left|\frac{\partial^{\beta}}{\partial \xi^{\beta}}(\tilde{A}(t, x, \xi)-A(t, x, \xi))\right| \leq C\langle\xi\rangle^{-\mu+\frac{1}{2}}(\chi(\langle\xi\rangle))^{1-\frac{1}{2}\langle\xi\rangle^{-|\beta|}}
$$

since $\chi(\langle\xi\rangle) \leq C\langle\xi\rangle^{\delta}$ and $\mu=\frac{\delta+2}{3}$,

$$
\leq C\langle\xi\rangle^{\frac{\delta-1}{6}}\langle\xi\rangle^{-|\beta|}
$$

and

$$
\tilde{A}(t, x, \xi) \in S_{1, \mu}^{0}
$$

Here we denote by $S_{\rho, \kappa}^{m}$ the set of all continuous $s(t, x, \xi)$ on $\boldsymbol{R}_{t} \times \boldsymbol{R}^{2 d}$ satisfying for any $\alpha$ and $\beta$,

$$
\left|\frac{\partial^{\alpha+\beta}}{\partial x^{\alpha} \partial \xi^{\beta}} S(t, x, \xi)\right| \leq C\langle\xi\rangle^{m-\rho|\beta|+\kappa|\alpha|}
$$

on $\boldsymbol{R}_{t} \times \boldsymbol{R}^{2 d}$. Since $\delta<1$, we have $\mu<1$. Thus the operator $\tilde{A}\left(t, x, D_{x}\right)$ is an $L^{2}$ bounded operator with the continuous parameter $t$. Since $\delta-1<0$, using the result of $\mathrm{M}$. Nagase [Na, Theorem 2 and 3], we see that the estimate (2.8) implies that $\tilde{A}\left(t, x, D_{x}\right)-A\left(t, x, D_{x}\right)$ is an $L^{2}$ bounded operator with the continuous parameter $t$. Hence the operator $A\left(t, x, D_{x}\right)$ is an $L^{2}$ bounded operator with the continuous parameter $t$. The proof of Proposition 2.1 is completed.

\section{§3. Carleman Estimates for First Order Operators}

We introduce two classes of symbols. We say that a smooth function $p(t, x, \xi)[\operatorname{resp} . p(x, \xi)]$ belongs to $S_{1 \cdot *}^{m}\left[\operatorname{resp} . S_{* \cdot *}^{0}\right]$ if we have, for any $\alpha_{0}, \alpha$ and $\beta$,

$$
\left|\frac{\partial^{\alpha_{0}}}{\partial t^{\alpha_{0}}} \frac{\partial^{\alpha}}{\partial x^{\alpha}} \frac{\partial^{\beta}}{\partial \xi^{\beta}} p(t, x, \xi)\right| \leq C\left(\langle\xi\rangle^{\frac{1}{2}}(\chi(\langle\xi\rangle))^{\frac{1}{2}}\right)^{\left|\alpha_{0}\right|+|\alpha|}\langle\xi\rangle^{m-|\beta|}
$$

on $\boldsymbol{R}_{t} \times \boldsymbol{R}^{2 d}$ [resp. if we have, for any $\alpha$ and $\beta$,

$$
\left|\frac{\partial^{\alpha+\beta}}{\partial x^{\alpha} \partial \xi^{\beta}} p(x, \xi)\right| \leq C_{\alpha, \beta}(\langle\xi\rangle \chi(\langle\xi\rangle))^{|\alpha|-|\beta|}
$$

on $\boldsymbol{R}^{2 d}$.] 
From (M.3) we see $S_{1 . *}^{m} \subset S_{1 . \frac{1+\delta}{2}}^{m}$. Since (M.4) implies that there exists two positive constant $c$ and $C$ such $^{2}$ that

$$
C^{-1} \leq \frac{\chi(\xi)}{\chi(\eta)} \leq C \quad \text { if }|\xi-\eta| \leq c|\xi|
$$

we see that $\Phi(\xi)=\langle\xi\rangle$ or $\Phi(\xi)=(\langle\xi\rangle \chi(\langle\xi\rangle))^{\frac{1}{2}}$ and $\varphi(\xi)=(\langle\xi\rangle \chi(\langle\xi\rangle))^{-\frac{1}{2}}$ are weights in a sense of R. Beals and C. Fefferman ([B-F] or see R. Beals [B] or H. Kumano-go [K, Ch. 7]) and then we can apply the results on the calculus of pseudodifferential operators given by R. Beals and C. Fefferman ( $[B-F]$, see also R. Beals [B] and H. Kumano-go [K. Ch. 7] for the calculus of the operators associated to $S_{1, *}^{m}$ or $S_{*, *}^{0}$.) Especially the pseudodifferential operators whose symbols are in $S_{1, *}^{0}$ or $S_{*, *}^{0}$ are $L^{2}$-bounded operators that are strongly continuous with respect to the parameter $t$. Next we remark that, since the estimate (M.3) implies $\chi(\langle\xi\rangle) \leq C\langle\xi\rangle^{\frac{1}{2}} \chi(\langle\xi\rangle)^{\frac{1}{2}}$, the symbol $\tilde{a}(t, x, \xi)$ defined in Section 2 belongs to $S_{1, *}^{0}$.

Now we define the weight function $\phi(s)$ with which we draw the Carleman estimate.

Since $\chi(s)$ is continuous and positive, the function $r(s)$ on $[1,+\infty)$ defined by

$$
r(s)=\int_{1}^{s} \frac{1}{s \chi(s)} d s
$$

is strictly increasing and (M.2) means $r(s) \rightarrow+\infty$ as $s \rightarrow+\infty$. Hence $r(s)$ has the $C^{1}$ inverse function $s(r)$ defined on $[0,+\infty)$. And we define $\phi(r)$ by

$$
\phi(r)=\int_{0}^{r} s(r) d r .
$$

By the definition we see that

$$
\phi^{\prime \prime}(r)=s \chi(s) \quad \text { if } \phi^{\prime}(r)=s .
$$

Since $\chi(s)$ is nondecreasing and $\phi^{\prime}(0)=1$, we see that

$$
\phi^{\prime \prime}(r) \geq \chi(1)
$$

Proposition 3.1. For real-valued symbols $p(t, x, \xi)$ and $q(t, x, \xi)$ in $S_{1, *}^{1}$ satisfying

$$
|q(t, x, \xi)| \geq C\langle\xi\rangle
$$




$$
\chi(\langle\xi\rangle)^{-1} \frac{\partial}{\partial t} p(t, x, \xi), \chi(\langle\xi\rangle)^{-1} \frac{\partial}{\partial t} q(t, x, \xi) \in S_{1, *}^{1}
$$

and for $1 \leq j \leq d$

$$
\chi(\langle\xi\rangle)^{-1} \frac{\partial}{\partial x_{j}} p(t, x, \xi), \chi(\langle\xi\rangle)^{-1} \frac{\partial}{\partial x_{j}} q(t, x, \xi) \in S_{1, *}^{1},
$$

we have the following. For any $t_{0}>0$ there exists a positive integer $n_{0}$ such that for any $v(t, x)$ in $C_{0}^{\infty}\left(\boldsymbol{R}^{d+1}\right)$ whose support is a compact subset of $\left[0, t_{0}\right] \times \boldsymbol{R}^{d}$, and for any $n \geq n_{0}$

$$
\begin{aligned}
& C_{0} n^{\frac{1}{2}(1-\delta)}\left\|e^{\frac{1}{h^{\phi}\left(n\left(2 t_{0}-t\right)\right)}}\left(\chi\left(\left\langle D_{x}\right\rangle\right)\left\langle D_{x}\right\rangle\right)^{\frac{1}{2}} v(t, x)\right\|^{2} \\
\leq & \left\|e^{\frac{1}{n} \phi\left(n\left(2 t_{0}-t\right)\right)}\left(D_{t}+p\left(t, x, D_{x}\right)-i q\left(t, x, D_{x}\right)\right) v(t, x)\right\|^{2},
\end{aligned}
$$

where the constant $C_{0}$ is independent of $n$ and, of course, of $v(t, x)$.

Proof. In this proof any positive constant $C$ is assumed to be independent of $n$. Since $p(t, x, \xi)$ and $q(t, x, \xi)$ are real-valued symbols in $S_{1, *}^{1}$,

$$
p_{a}\left(t, x, D_{x}\right)=\frac{1}{2}\left(p\left(t, x, D_{x}\right)-p^{*}\left(t, x, D_{x}\right)\right)
$$

and

$$
q_{a}\left(t, x, D_{x}\right)=\frac{1}{2}\left(q\left(t, x, D_{x}\right)-q^{*}\left(t, x, D_{x}\right)\right)
$$

are pseudodifferential operators whose symbols $p_{a}(t, x, \xi)$ and $q_{a}(t, x, \xi)$ satisfy

$$
\begin{aligned}
& p_{a}(t, x, \xi)(\chi(\langle\xi\rangle)\langle\xi\rangle)^{-\frac{1}{2}} \in S_{1, *}^{0} \\
& q_{a}(t, x, \xi)(\chi(\langle\xi\rangle)\langle\xi\rangle)^{-\frac{1}{2}} \in S_{1, *}^{0},
\end{aligned}
$$

where $p^{*}\left(t, x, D_{x}\right)$ [resp. $\left.q^{*}\left(t, x, D_{x}\right)\right]$ is the formal adjoint of $p\left(t, x, D_{x}\right)$ [resp. $\left.q\left(t, x, D_{x}\right)\right]$ with respect to the inner product on $L^{2}\left(\boldsymbol{R}_{x}^{d}\right)$.

Hence for any $f(x) \in C_{0}^{\infty}\left(\boldsymbol{R}^{d}\right)$

$$
\begin{aligned}
& \left\|p_{a}\left(t, x, D_{x}\right) f(x)\right\| \leq C\left\|\left(\chi\left(\left\langle D_{x}\right\rangle\right)\left\langle D_{x}\right\rangle\right)^{\frac{1}{2}} f(x)\right\| \\
& \left\|q_{a}\left(t, x, D_{x}\right) f(x)\right\| \leq C\left\|\left(\chi\left(\left\langle D_{x}\right\rangle\right)\left\langle D_{x}\right\rangle\right)^{\frac{1}{2}} f(x)\right\| .
\end{aligned}
$$


By using the operators

$$
\begin{aligned}
& p_{s}\left(t, x, D_{x}\right)=p\left(t, x, D_{x}\right)-p_{a}\left(t, x, D_{x}\right) \\
& q_{s}\left(t, x, D_{x}\right)=q\left(t, x, D_{x}\right)-q_{a}\left(t, x, D_{x}\right)
\end{aligned}
$$

we define the operator $L$ by

$$
L v(t, x)=\left(\frac{\partial}{\partial t}+i p_{s}\left(t, x, D_{x}\right)+q_{s}\left(t, x, D_{x}\right)\right) v(t, x)
$$

We set

$$
w(t, x)=e^{\frac{1}{n} \phi\left(n\left(2 t_{0}-t\right)\right)} v(t, x) .
$$

Then, since $e^{\frac{1}{n} \phi\left(n\left(2 t_{0}-t\right)\right)} L v(t, x)=\left(L+\phi^{\prime}\left(n\left(2 t_{0}-t\right)\right) w(t, x)\right.$,

$$
\begin{aligned}
& \left\|e^{\frac{1}{n} \phi\left(n\left(2 t_{0}-t\right)\right)} L v(t, x)\right\|^{2} \\
= & \left\|\left(\frac{\partial}{\partial t}+i p_{s}\left(t, x, D_{x}\right)\right) w(t, x)\right\|^{2}+\left\|\left(\phi^{\prime}\left(n\left(2 t_{0}-t\right)\right)+q_{s}\left(t, x, D_{x}\right)\right) w(t, x)\right\|^{2} \\
& +2 \mathfrak{R}\left(\left(\frac{\partial}{\partial t}+i p_{s}\left(t, x, D_{x}\right)\right) w(t, x),\left(\phi^{\prime}\left(n\left(2 t_{0}-t\right)\right)+q_{s}\left(t, x, D_{x}\right)\right) w(t, x)\right) .
\end{aligned}
$$

Since $\left(p_{s}\left(t, x, D_{x}\right)\right)^{*}=p_{s}\left(t, x, D_{x}\right)$ and $\left(q_{s}\left(t, x, D_{x}\right)\right)^{*}=q_{s}\left(t, x, D_{x}\right)$, the third term of the right hand side is equal to

$$
\begin{aligned}
& \frac{\partial}{\partial t}\left(\left(\phi^{\prime}\left(n\left(2 t_{0}-t\right)\right)+q_{s}\left(t, x, D_{x}\right)\right) w(t, x), w(t, x)\right) \\
& \quad+\left(\left(n \phi^{\prime \prime}\left(n\left(2 t_{0}-t\right)\right)-\frac{\partial}{\partial t} q_{s}\left(t, x, D_{x}\right)\right) w(t, x), w(t, x)\right) \\
& \quad+i\left(\left[q_{s}\left(t, x, D_{x}\right), p_{s}\left(t, x, D_{x}\right)\right] w(t, x), w(t, x)\right) .
\end{aligned}
$$

From the (3.4), (3.5), (3.6) and (3.8) we see that

$$
\frac{\frac{\partial}{\partial t} q_{s}\left(t, x, D_{x}\right)}{\langle\xi\rangle \chi(\langle\xi\rangle)} \in S_{1, *}^{0}
$$


and

$$
\frac{\frac{\partial}{\partial \xi_{j}} q_{s}(t, x, \xi) \frac{\partial}{\partial x_{j}} p_{s}(t, x, \xi)-\frac{\partial}{\partial \xi_{j}} p_{s}(t, x, \xi) \frac{\partial}{\partial x_{j}} q_{s}(t, x, \xi)}{\langle\xi\rangle \chi(\langle\zeta \zeta\rangle)} \in S_{1, *}^{0},
$$

which imply that

$$
\begin{aligned}
\left.\mid\left(\frac{\partial}{\partial t} q_{s}\left(t, x, D_{x}\right)\right) w(t, x), w(t, x)\right)|+|\left(\left[q_{s}\left(t, x, D_{x}\right), p_{s}\left(t, x, D_{x}\right)\right] w(t, x), w(t, x)\right) \mid & \\
& \leq C\left\|\left(\left\langle D_{x}\right\rangle \chi\left(\left\langle D_{x}\right\rangle\right)\right)^{\frac{1}{2}} w(t, x)\right\|^{2}
\end{aligned}
$$

Therefore we have, since $w(t, x)$ vanishes at $t=0$ and $t=t_{0}$,

$$
\begin{aligned}
& \int_{0}^{t_{0}}\left\|e^{\frac{1}{n} \phi\left(n\left(2 t_{0}-t\right)\right)} L v(t, x)\right\|^{2} d t \\
\geq & \int_{0}^{t_{0}}\left(\left\|\left(\phi^{\prime}\left(n\left(2 t_{0}-t\right)\right)+q_{s}\left(t, x, D_{x}\right)\right) w(t, x)\right\|^{2}\right. \\
& \left.+n \phi^{\prime \prime}\left(n\left(2 t_{0}-t\right)\right)\|w(t, x)\|^{2}\right) d t \\
& -C \int_{0}^{t_{0}}\left\|\left(\left\langle D_{x}\right\rangle \chi\left(\left\langle D_{x}\right\rangle\right)\right)^{\frac{1}{2}} w(t, x)\right\|^{2} d t
\end{aligned}
$$

Thus in order to finish the proof of Proposition 3.1 we have only to show the following estimate: for $t \in\left[0, t_{0}\right]$

$$
(V w(t, x), w(t, x)) \geq\left(C_{1} n^{\frac{1}{2}(1-\delta)}-C_{2}\right)\left\|\left(\left\langle D_{x}\right\rangle \chi\left(\left\langle D_{x}\right\rangle\right)\right)^{\frac{1}{2}} w(t, x)\right\|^{2},
$$

where $V=\left(\phi^{\prime}\left(n\left(2 t_{0}-t\right)\right)+q_{s}\left(t, x, D_{x}\right)\right)^{2}+n \phi^{\prime \prime}\left(n\left(2 t_{0}-t\right)\right)$.

Indeed it follows from (3.11) and (3.12) that

$$
\begin{gathered}
\int_{0}^{t_{0}}\left\|e^{\frac{1}{n} \phi\left(n\left(2 t_{0}-t\right)\right)} L v(t, x)\right\|^{2} d t \\
\geq\left(C_{1} n^{\frac{1}{2}(1-\delta)}-C_{2}\right) \int_{0}^{t_{0}}\left\|\left(\left\langle D_{x}\right\rangle \chi\left(\left\langle D_{x}\right\rangle\right)\right)^{\frac{1}{2}} w(t, x)\right\|^{2} d t,
\end{gathered}
$$

from which, noting (3.7) and

$$
D_{t}+p\left(t, x, D_{x}\right)-i q\left(t, x, D_{x}\right)=\frac{1}{i}\left\{L+i p_{a}\left(t, x, D_{x}\right)+q_{a}\left(t, x, D_{x}\right)\right\},
$$


we obtain the assertion of Proposition 3.1 for some $n_{0}$.

Set

$$
Q(t, x, \xi)=\sqrt{n \phi^{\prime \prime}\left(n\left(2 t_{0}-t\right)\right)}-i\left(\phi^{\prime}\left(n\left(2 t_{0}-t\right)\right)+q(t, x, \xi)\right) .
$$

Noting (3.7), (3.8) and

$$
(V w(t, x), w(t, x))=\left\|\left(\sqrt{n \phi^{\prime \prime}\left(n\left(2 t_{0}-t\right)\right)}-i\left(\phi^{\prime}\left(n\left(2 t_{0}-t\right)\right)+q_{s}\left(t, x, D_{x}\right)\right)\right) w(t, x)\right\|^{2},
$$

we see that (3.12) is equivalent to

$$
\left\|Q\left(t, x, D_{x}\right) w(t, x)\right\| \geq\left(C_{1} n^{\frac{1}{4}(1-\delta)}-C_{2}\right)\left\|\left(\left\langle D_{x}\right\rangle \chi\left(\left\langle D_{x}\right\rangle\right)\right)^{\frac{1}{2}} w(t, x)\right\| .
$$

In order to obtain the estimate (3.13), first we show that for $t \in\left[0, t_{0}\right]$

$$
|Q(t, x, \xi)| \geq C n^{\frac{1}{4}(1-\delta)}(\chi(\langle\xi\rangle)\langle\xi\rangle)^{\frac{1}{2}}
$$

and that, setting

$$
E_{n}(t, x, \xi)=\frac{n^{\frac{1}{4}(1-\delta)}(\chi(\langle\xi\rangle)\langle\xi\rangle)^{\frac{1}{2}}}{Q(t, x, \xi)}
$$

$$
\left\{E_{n}(t, x, \xi)\right\}_{n \geq 1, t \in\left[0, t_{0}\right]} \text { is a bounded family in } S_{*, *}^{0} .
$$

Since $q(t, x, \xi) \in S_{1, *}^{1}$ is real-valued and satisfies (3.3), we see that there exist two positive constants $C_{1}$ and $C_{2}$ such that if $q(t, x, \xi) \geq 0$,

$$
C_{1}\langle\xi\rangle \leq q(t, x, \xi) \leq C_{2}\langle\xi\rangle
$$

and if $q(t, x, \xi)<0$,

$$
C_{1}\langle\xi\rangle \leq-q(t, x, \xi) \leq C_{2}\langle\xi\rangle
$$

In the case where, using the constants above,

$$
\frac{1}{2} C_{1}\langle\xi\rangle \leq \phi^{\prime}\left(n\left(2 t_{0}-t\right)\right) \leq 2 C_{2}\langle\xi\rangle
$$

we see from (3.1) and the monotonicity of $\chi(s)$, that

$$
\phi^{\prime \prime}\left(n\left(2 t_{0}-t\right)\right) \geq \frac{1}{2} C_{1}\langle\xi\rangle \chi\left(\frac{1}{2} C_{1}\langle\xi\rangle\right)
$$


from (M.1)

$$
\geq C\langle\xi\rangle \chi(\langle\xi\rangle)
$$

Hence

$$
|Q(t, x, \xi)| \geq C n^{\frac{1}{2}}(\langle\xi\rangle \chi(\langle\xi\rangle))^{\frac{1}{2}},
$$

which implies (3.14).

In the case where

$$
\frac{1}{2} C_{1}\langle\xi\rangle \geq \phi^{\prime}\left(n\left(2 t_{0}-t\right)\right) \quad \text { or } \quad \phi^{\prime}\left(n\left(2 t_{0}-t\right)\right) \geq 2 C_{2}\langle\xi\rangle
$$

since $\phi^{\prime}(r) \geq 1$ for $r \geq 0$, we obtain from (3.16) and (3.17)

$$
\left|\left(\phi^{\prime}\left(n\left(2 t_{0}-t\right)\right)+q(t, x, \xi)\right)\right| \geq C\langle\xi\rangle .
$$

Hence in this case, since $\phi^{\prime \prime}(r) \geq \chi(1)$ for $r \geq 0$ (see (3.2)),

$$
\begin{aligned}
|Q(t, x, \xi)| & \geq C\left(n^{\frac{1}{2}}+\langle\xi\rangle\right) \\
& \geq C\left(n^{\frac{1}{2}}\right)^{1-\frac{1}{2}(\delta+1)}\langle\xi\rangle^{\frac{1}{2}(\delta+1)},
\end{aligned}
$$

from (M.3)

$$
\geq C n^{\frac{1}{4}(1-\delta)}(\chi(\langle\xi\rangle)\langle\xi\rangle)^{\frac{1}{2}}
$$

which shows (3.14).

Next if $|\alpha|>0$, then

$$
\begin{aligned}
\left|\frac{\partial^{\alpha+\beta}}{\partial x^{\alpha} \partial \xi^{\beta}} Q(t, x, \xi)\right| & =\left|\frac{\partial^{\alpha+\beta}}{\partial x^{\alpha} \partial \xi^{\beta}} q(t, x, \xi)\right| \\
& \leq C\left(\langle\xi\rangle \chi(\langle\xi\rangle)^{\frac{1}{2}(|\alpha|+1)}\langle\xi\rangle^{-|\beta|}\right.
\end{aligned}
$$

Hence, taking (3.14) into account, if $|\alpha|>0$,

$$
\left|\frac{\frac{\partial^{\alpha+\beta}}{\partial x^{\alpha} \delta \xi^{\beta}} Q(t, x, \xi)}{Q(t, x, \xi)}\right| \leq C(\langle\xi\rangle \chi(\langle\xi\rangle))^{\frac{1}{2}|\alpha|}\langle\xi\rangle-|\beta| .
$$

Similarly for $|\beta|>0$, since $\left|\frac{\partial^{\beta}}{\partial \xi^{\beta}} Q(t, x, \xi)\right|=\left|\frac{\partial^{\beta}}{\partial \xi^{\beta}} q(t, x, \xi)\right|$, 


$$
\left|\frac{\frac{\partial^{\beta}}{\partial \xi^{\beta}} Q(t, x, \xi)}{Q(t, x, \xi)}\right| \leq C(\langle\xi\rangle \chi(\langle\xi\rangle))^{-\frac{1}{2}\langle\xi\rangle^{1-|\beta|}} .
$$

The assertion (3.15) follows from (3.19) and (3.20). Thus (3.14) and (3.15) are proved.

It follows from (3.15) that for $t \in\left[0, t_{0}\right]$

$$
\left\|E_{n}\left(t, x, D_{x}\right) f(x)\right\| \leq C\|f(x)\| .
$$

We define the operator $R_{n}\left(t, x, D_{x}\right)$ by

$$
R_{n}\left(t, x, D_{x}\right)=n^{\frac{1}{4}(1-\delta)}\left\langle D_{x}\right\rangle^{\frac{1}{2}}\left(\chi\left(\left\langle D_{x}\right\rangle\right)\right)^{\frac{1}{2}}-E_{n}\left(t, x, D_{x}\right) Q\left(t, x, D_{x}\right)
$$

and let $R_{n}(t, x, \xi)$ be its symbol. Since the symbol $R_{n}(t, x, \xi)$ is given by the following oscillatory integral

$$
\sum_{j=1}^{d} \int_{0}^{1} d \theta \frac{1}{(2 \pi)^{d}} \int_{R^{2 d}} e^{-i\langle y, \eta\rangle}(-1)^{\frac{1}{2}} \frac{\partial}{\partial \xi_{j}} E_{n}(t, x, \xi+\theta \eta) \frac{\partial}{\partial x_{j}} Q(t, x+y, \xi) d \eta d y
$$

then it follows from (3.15) and (3.18) that

$$
\left\{\frac{R_{n}(t, x, \xi)}{(\langle\xi\rangle \chi(\langle\xi\rangle))^{\frac{1}{2}}}\right\}_{n \geq 1, t \in\left[0, t_{0}\right]}
$$

is a bounded family in $S_{* \cdot *}^{0}$ (see [B-F, §2, Theorem 1 and Lemma 2]). Thus we obtain, for $t \in\left[0, t_{0}\right]$,

$$
\left\|R_{n}\left(t, x, D_{x}\right) f(x)\right\| \leq C\left\|\left\langle D_{x}\right\rangle^{\frac{1}{2}}\left(\chi\left(\left\langle D_{x}\right\rangle\right)\right)^{\frac{1}{2}} f(x)\right\| .
$$

Since

$$
\begin{aligned}
& \left\|n^{\frac{1}{4}(1-\delta)}\left\langle D_{x}\right\rangle^{\frac{1}{2}}\left(\chi\left(\left\langle D_{x}\right\rangle\right)\right)^{\frac{1}{2}} w(t, x)\right\| \\
\leq & \left\|E_{n}\left(t, x, D_{x}\right) Q\left(t, x, D_{x}\right) w(t, x)\right\|+\left\|R_{n}\left(t, x, D_{x}\right) w(t, x)\right\|,
\end{aligned}
$$

the desired estimate (3.13) follows from (3.21) and (3.23). This completes the proof of Proposition 3.1 


\section{§4. Proof of Theorem 1.2.}

First we regularize the principal part of the operator:

$$
e(t, x, \tau, \xi)=\tau^{2}+\sum_{j=1}^{d} 2 a_{0, j}(t, x) \tau \xi_{j}+\sum_{j . k=1}^{d} a_{j, k}(t, x) \xi_{j} \xi_{k} .
$$

Set

$$
b_{k, j}(t, x)=a_{j, k}(t, x)-a_{0, j}(t, x) a_{0, k}(t, x) .
$$

Then we see that

$$
e(t, x, \tau, \xi)=\left(\tau+\sum_{j=1}^{d} a_{0, j}(t, x) \xi_{j}\right)^{2}+\sum_{j, k=1}^{d} b_{j, k}(t, x) \xi_{j} \xi_{k}
$$

and from the ellipticity

$$
\sum_{j, k=1}^{d} b_{j, k}(t, x) \xi_{j} \xi_{k} \geq C|\xi|^{2}
$$

We denote by $\tilde{a}_{0 . j}(t, x, \xi)$ [resp. $\left.\tilde{b}_{j . k}(t, x, \xi)\right]$ the regularization of $a_{0, j}(t, x)$ [resp. $\left.b_{j, k}(t, x)\right]$ defined in Section 2.

Set

$$
p(t, x, \xi)=\sum_{j=1}^{d} \tilde{a}_{0, j}(t, x, \xi) \xi_{j}
$$

and

$$
b(t, x, \xi)=\sum_{j, k=1}^{d} \tilde{b}_{j, k}(t, x, \xi) \xi_{j} \xi_{k} .
$$

Then the definition of $b_{j, k}(t, x)$ and (4.2) imply

$$
b(t, x, \xi) \geq C|\xi|^{2} .
$$

Here we introduce a notation. Let $m(\xi)$ be a continuous function on $\boldsymbol{R}^{d}$ with a polynomial growth. We say that an operator $P(t)$ on $C_{0}^{\infty}\left(\boldsymbol{R}^{d}\right)$ with a parameter $t \in\left[0, t_{0}\right]$ belongs to $\mathscr{L}\{m(\xi)\}$ if we have for any $f(x) \in C_{0}^{\infty}\left(\boldsymbol{R}^{d}\right)$ and any $t \in\left[0, t_{0}\right]$

$$
\|P(t) f(x)\| \leq C\left\|m\left(D_{x}\right) f(x)\right\| .
$$




\section{Lemma 4.1.}

$$
\begin{aligned}
& \left(D_{t}+p\left(t, x, D_{x}\right)\right)^{2}+b\left(t, x, D_{x}\right) \\
= & D_{t}^{2}+2 \sum_{j=1}^{d} a_{0 . j}(t, x) D_{t} D_{x_{j}}+\sum_{j, k=1}^{d} a_{j, k}(t, x) D_{x_{j}} D_{x_{k}}+R_{0}(t) D_{t}+R_{1}(t)
\end{aligned}
$$

where $R_{j}(t) \in \mathscr{L}\left\{\langle\xi\rangle^{\frac{1+2 i}{2}}(\chi(\langle\xi\rangle))^{\frac{1}{2}}\right\}$.

Proof. Since $\tilde{a}_{0 . j}(t, x, \xi) \in S_{1, *}^{0}$,

$$
\left[D_{t}, p\left(t, x, D_{x}\right)\right]
$$

and

$$
p\left(t, x, D_{x}\right)^{2}-\sum_{j . k=1}^{d} \tilde{a}_{0, j}\left(t, x, D_{x}\right) \tilde{a}_{0, k}\left(t, x, D_{x}\right) D_{x_{j}} D_{x_{k}}
$$

belong to $\mathscr{L}\left\{\langle\xi\rangle^{\frac{3}{2}}(\chi(\langle\xi\rangle))^{\frac{1}{2}}\right\}$. Since, thanks to Proposition 2.1, $\tilde{a}_{0, j}\left(t, x, D_{x}\right)$ $-a_{0, j}(t, x) \in \mathscr{L}\left\{\langle\xi\rangle^{-\frac{1}{2}}(\chi(\langle\xi\rangle))^{\frac{1}{2}}\right\}$, we see that

$$
\sum_{j=1}^{d} a_{0 . j}(t, x) D_{x_{j}}-p\left(t, x, D_{x}\right) \in \mathscr{L}\left\{\langle\xi\rangle^{\frac{1}{2}}(\chi(\langle\xi\rangle))^{\frac{1}{2}}\right\}
$$

and that

$$
\tilde{a}_{0, j}\left(t, x, D_{x}\right) \tilde{a}_{0, k}\left(t, x, D_{x}\right) D_{x_{j}} D_{x_{k}}-a_{0 . j}(t, x) a_{0, k}(t, x) D_{x_{j}} D_{x_{k}}
$$

belongs to $\mathscr{L}\left\{\langle\xi\rangle^{\frac{3}{2}}(\chi(\langle\xi\rangle))^{\frac{1}{2}}\right\}$. Therefore we obtain that the following operator can be written as $r_{0}(t) D_{t}+r_{1}(t)$ with $r_{j}(t) \in \mathscr{L}\left\{\langle\xi\rangle^{\frac{1+2 i}{2}}(\chi(\langle\xi\rangle))^{\frac{1}{2}}\right\}$

$$
D_{t}^{2}+\sum_{j=1}^{d} 2 a_{0, j}(t, x) D_{t} D_{x_{j}}+\sum_{j, k=1}^{d} a_{0, j}(t, x) a_{0, k}(t, x) D_{x_{j}} D_{x_{k}}-\left(D_{t}+p\left(t, x, D_{x}\right)\right)^{2}
$$

Similarly, since

$$
\tilde{b}_{j, k}\left(t, x, D_{x}\right)-b_{j, k}(t, x) \in \mathscr{L}\left\{\langle\xi\rangle^{-\frac{1}{2}}(\chi(\langle\xi\rangle))^{\frac{1}{2}}\right\},
$$

we see

$$
b\left(t, x, D_{x}\right)-\sum_{j, k=1}^{d} b_{j . k}(t, x) D_{x_{j}} D_{x_{k}} \in \mathscr{L}\left\{\langle\xi\rangle^{\frac{3}{2}}(\chi(\langle\xi\rangle))^{\frac{1}{2}}\right\}
$$


Hence we obtain the assertion of Lemma 4.1.

Set, with a positive constant $l \geq 1$

$$
q_{l}(t, x, \xi)=\sqrt{b(t, x, \xi)+l^{2}}
$$

From Proposition 2.1, $b(t, x, \xi) \in S_{1 . *}^{2}$ and (4.3), we see that $q_{l}(t, x, \xi)$ satisfies the assumption on $q(t, x, \xi)$ of Proposition 3.1 and that

$$
q_{l}\left(t, x, D_{x}\right) q_{l}\left(t, x, D_{x}\right)-b\left(t, x, D_{x}\right) \in \mathscr{L}\left\{\langle\xi\rangle^{\frac{3}{2}}(\chi(\langle\xi\rangle))^{\frac{1}{2}}\right\} .
$$

Furthermore choosing $l$ large enough, we see that there exists $o(t, x, \xi) \in S_{1, \delta^{0}}^{-1}$ where $\delta_{0}=\frac{1+\delta}{2}$ such that

$$
o\left(t, x, D_{x}\right) q_{l}\left(t, x, D_{x}\right)=1 .
$$

Indeed, since $q_{l}(t, x, \xi) \geq C(|\xi|+l)$ and $b(t, x, \xi) \in S_{1, \delta_{0}}^{2}$ we obtain for $|\alpha|+|\beta|>0$

$$
\left|\frac{\partial^{\alpha+\beta}}{\partial x^{\alpha} \partial \xi^{\beta}} q_{l}(t, x, \xi)\right| \leq C_{\alpha, \beta}(|\xi|+l)^{-1}\langle\xi\rangle^{2+\delta_{0}|\alpha|-|\beta|}
$$

with the constant $C_{\alpha, \beta}$ that is independent of $l \geq 1$. Hence $\left\{\frac{\partial}{\partial x_{j}} q_{l}(t, x, \xi) l^{\left(1-\delta_{0}\right)} \mid 1\right.$ $\leq j \leq d, l \geq 1\}$ is bounded in $S_{1, \delta_{0}}^{2}$. Set $q_{l}^{-1}(t, x, \xi)=\frac{1}{q_{l}(t, x, \xi)}$ and $r_{l}(t, x, \xi)$ the symbol of the operator

$$
q_{l}^{-1}\left(t, x, D_{x}\right) q_{l}\left(t, x, D_{x}\right)-I .
$$

Then, since $\left\{q_{l}^{-1}(t, x, \xi) \mid l \geq 1\right\}$ is bounded in $S_{1, \delta_{0}}^{-1},\left\{r_{l}(t, x, \xi) l^{1-\delta_{0}} \mid l \geq 1\right\}$ becomes a bounded set in $S_{1, \delta_{0}}^{0}$ (see H. Kumano-go ([K, Ch. 3, §3, Theorem 3.1]). Hence for large $l$, the operator $I+r_{l}\left(t, x, D_{x}\right)$ has the inverse $I\left(t, x, D_{x}\right)$ whose symbol $I(t, x, \xi)$ belongs to $S_{1 . \delta_{0}}^{0}$ (see H. Kumano-go ([K, Appendix, Theorem I.1]). Therefore $I\left(t, x, D_{x}\right) q_{l}^{-1}\left(t, x, D_{x}\right)$ is a desired operator satisfying (4.5). Since we consider $q_{l}(t, x, \xi)$ with one fixed $l$ for which (4.5) is valid, in the following we write $q(t, x, \cdot)$ in the place of $q_{l}(t, x, \cdot)$.

Now using

$$
L_{1}=D_{t}+p\left(t, x, D_{x}\right)-i q\left(t, x, D_{x}\right)
$$


and

$$
L_{2}=D_{t}+p\left(t, x, D_{x}\right)+i q\left(t, x, D_{x}\right)
$$

we can rewrite the operator $E\left(t, x, D_{t}, D_{x}\right)$ in the following way. Since $p(t, x, \xi)$ and $q(t, x, \xi) \in S_{1, *}^{1}$,

$$
\left(D_{t}+p\left(t, x, D_{x}\right)\right)^{2}+q\left(t, x, D_{x}\right)^{2}-L_{1} L_{2} \in \mathscr{L}\left\{\langle\xi\rangle^{\frac{3}{2}}(\chi(\langle\xi\rangle))^{\frac{1}{2}}\right\} .
$$

Since $D_{t}+p\left(t, x, D_{x}\right)=\frac{1}{2}\left(L_{1}+L_{2}\right), q\left(t, x, D_{x}\right)=\frac{1}{2 i}\left(L_{2}-L_{1}\right)$ and the coefficients $a_{j}(t, x) c(t, x)$ of $E\left(t, x, D_{t}, D_{x}\right)$ are bounded, it follows from Lemma 4.1, (4.4), (4.5) and (4.6) that there exist $Q_{1,1}$ and $Q_{1,2}$ in $\mathscr{L}\left\{\langle\xi\rangle^{\frac{1}{2}}(\chi(\langle\xi\rangle))^{\frac{1}{2}}\right\}$ such that

$$
L_{1} L_{2}=E\left(t, x, D_{t}, D_{x}\right)+\sum_{j=1,2} Q_{1, j} L_{j}
$$

Similarly we see that there exist $Q_{2,1}$ and $Q_{2,2}$ in $\mathscr{L}\left\{\langle\xi\rangle^{\frac{1}{2}}(\chi(\langle\xi\rangle))^{\frac{1}{2}}\right\}$ such that

$$
L_{2} L_{1}=E\left(t, x, D_{t}, D_{x}\right)+\sum_{j=1.2} Q_{2, j} L_{j}
$$

Finally let $u(t, x)$ and $f(t, x)$ be those of Theorem 1.2. Set for $j=1,2$

$$
v_{j}(t, x)=L_{3-j} u(t, x) \text {. }
$$

Then we see that for $j=1,2 v_{j}(t, x) \in H^{1}\left(\boldsymbol{R}^{d+1}\right)$ and the support of $v_{j}(t, x)$ is in $\left[0, \frac{2}{3} t_{0}\right] \times \boldsymbol{R}^{d}$. Proposition 3.1 and the density argument give the following; there exists a positive $n_{0}$ such that for any $n \geq n_{0}$

$$
n^{\frac{1-\delta}{4}}\left\|e^{\phi_{n}(t)}\left\langle D_{x}\right\rangle^{\frac{1}{2}}\left(\chi\left(\left\langle D_{x}\right\rangle\right)\right)^{\frac{1}{2}} v_{j}(t, x)\right\| \leq C\left\|e^{\phi_{n}(t)} L_{j} v_{j}(t, x)\right\|,
$$

where $\phi_{n}(t)=\frac{1}{n} \phi\left(n\left(2 t_{0}-t\right)\right)$.

On the other hand from (4.7) we obtain

$$
L_{1} v_{1}(t, x)=f(t, x)+\sum_{j=1,2} Q_{1,3-j} v_{j}(t, x)
$$

which implies

$$
\begin{aligned}
& \left\|e^{\phi_{n}(t)} L_{1} v_{1}(t, x)\right\| \\
& \leq\left\|e^{\phi_{n}(t)} f(t, x)\right\|+C \sum_{j=1,2}\left\|e^{\phi_{n}(t)}\left\langle D_{x}\right\rangle^{\frac{1}{2}}\left(\chi\left(\left\langle D_{x}\right\rangle\right)\right)^{\frac{1}{2}} v_{j}(t, x)\right\| .
\end{aligned}
$$


Similarly from (4.8) we get

$$
\begin{aligned}
& \left\|e^{\phi_{n}(t)} L_{2} v_{2}(t, x)\right\| \\
& \leq\left\|e^{\phi_{n}(t)} f(t, x)\right\| \mid+C \sum_{j=1,2}\left\|e^{\phi_{n}(t)}\left\langle D_{x}\right\rangle^{\frac{1}{2}}\left(\chi\left(\left\langle D_{x}\right\rangle\right)\right)^{\frac{1}{2}} v_{j}(t, x)\right\| .
\end{aligned}
$$

Therefore, by choosing a larger $n_{0}$ if necessary, we obtain from (4.9), (4.10) and (4.11)

$$
\begin{gathered}
n^{\frac{1-\delta}{4}}\left(\left\|e^{\phi_{n}(t)}\left\langle D_{x}\right\rangle^{\frac{1}{2}}\left(\chi\left(\left\langle D_{x}\right\rangle\right)\right)^{\frac{1}{2}} v_{1}(t, x)\right\|\right. \\
\left.+\left\|e^{\phi_{n}(t)}\left\langle D_{x}\right\rangle^{\frac{1}{2}}\left(\chi\left(\left\langle D_{x}\right\rangle\right)\right)^{\frac{1}{2}} v_{2}(t, x)\right\|\right) \\
\leq C\left\|e^{\phi_{n}(t)} f(t, x)\right\|
\end{gathered}
$$

for $n \geq n_{0}$.

Since $\phi_{n}(t)$ is decreasing and $f(t, x)=0$ for $t \leq \frac{1}{2} t_{0}$, we get from (4.12)

$$
\begin{array}{r}
\sum_{j=1,2} n^{\frac{1-\delta}{2}} e^{2 \phi_{n}\left(\frac{1}{3} t_{0}\right)} \int_{0}^{\frac{1}{3} t_{0}}\left\|\left\langle D_{x}\right\rangle^{\frac{1}{2}}\left(\chi\left(\left\langle D_{x}\right\rangle\right)\right)^{\frac{1}{2}} v_{j}(t, x)\right\|^{2} d t \\
\leq C e^{2 \phi_{n}\left(\frac{1}{2} t_{0}\right)} \int_{\frac{1}{2} t_{0}}^{t_{0}}\|f(t, x)\|^{2} d t
\end{array}
$$

Therefore for $n \geq n_{0}$

$$
\begin{aligned}
& \sum_{j=1,2} \int_{0}^{\frac{1}{3} t_{0}} \|\left\langle D_{x}\right\rangle^{\frac{1}{2}}\left(\chi\left(\left\langle D_{x}\right\rangle\right)\right)^{\frac{1}{2}} v_{j}(t, x) \|^{2} d t \\
& \leq C n^{-\frac{1-\delta}{2}} e^{2\left(\phi_{n}\left(\frac{1}{2} t_{0}\right)-\phi_{n}\left(\frac{1}{3} t_{0}\right)\right)} \int_{\frac{1}{2} t_{0}}^{t_{0}}\|f(t, x)\|^{2} d t .
\end{aligned}
$$

Since $\phi_{n}(t)$ is decreasing, $\phi_{n}\left(\frac{1}{2} t_{0}\right)-\phi_{n}\left(\frac{1}{3} t_{0}\right)$ is negative. Then, since $\delta<1$, as $n \rightarrow+\infty$, the right hand side of (4.13) tends to zero. Hence the left hand side of (4.13) is equal to zero. Thus we see that $v_{1}(t, x)=0$ and $v_{2}(t, x)=0$ for $t<\frac{1}{3} t_{0}$. Hence, from $u(t, x)=\frac{1}{2 i} o\left(t, x, D_{x}\right)\left(v_{1}(t, x)-v_{2}(t, x)\right)$, we get $u(t, x)=0$ for $t<\frac{1}{3} t_{0}$. The proof of Theorem 1.2 is completed.

Remark. By the definiton of $\phi_{n}(t)$, we see that 


$$
\phi_{n}\left(\frac{1}{3} t_{0}\right)-\phi_{n}\left(\frac{1}{2} t_{0}\right)=\frac{1}{n} \int_{\frac{3}{2} t_{0} n}^{\frac{5}{3} t_{0} n} \phi^{\prime}(r) d r
$$

Since $t_{0}>0$ and $\phi^{\prime}(r)=s(r) \rightarrow+\infty$ as $r \rightarrow+\infty$, we see that the right hand side tends to $+\infty$ as $n \rightarrow+\infty$.

\section{References}

[B] Beals, R., A general calculus of pseudodifferential operators, Duke Math. J., 42 (1975), $1-42$.

[B-C] Bahouri, H. and Chemin, J.-Y., Equations de transport relatives à des champs de vecteurs non-lipshitzien et de mécanique des fluides, Arch. Rational Mech. Anal., 127 (1994), 159-181.

[B-F] Beals, R. and Fefferman, C., Spatially Inhomgeneous Pseudodifferential Operators, I, Com. Pure Appl. Math., 27 (1974), 1-24.

[C-L] Colombini, F. and Lerner, N., Hyperbolic Operators with Non-Lipschitz Coefficients, Duke Math. J., 177 (1995), 657-698.

[H1] Hörmander, L., On the uniqueness of Cauchy problem II, Math. Scand., 7 (1959), 177-190.

[H2] , The Analysis of Linear Partial Differential Operators III, Springer-Verlag, Berlin, 1985.

[K] Kumano-go, H., Pseudo-Differential Operators, The MIT Press, Cambridge, 1981.

[Na] Nagase, M., The $L^{p}$-boundedness of Pseudo-Differential Operators with Non-Regular Symbols, Comm. Partial Differential Equations, 2 (1977), 1045-1061.

[Ni] Nirenberg, L., Lectures on linear partial differential equations, Amer. Math. Soc. Regional Conf. in Math., 17, 1972.

[P] Pliś, A., On Non-Uniqueness in Cauchy Problem for an Elliptic Second Order Differential Equation, Bull. Pol. Acad. Sci. Math., 11 (1963), 95-100.

[Z] Zuily, C., Uniqueness and Non-Uniqueness in the Cauchy Problem, Birkhäuser, Boston, 1983. 